\title{
Hironaka's characteristic polygon and effective resolution of surfaces
}

\author{
Piedra, R.* $\quad$ Tornero, J.M. ${ }^{\dagger}$
}

August, 2005

\begin{abstract}
Hironaka's concept of characteristic polyhedron of a singularity has been one of the most powerful and fruitful ideas of the last decades in singularity theory. In fact, since then combinatorics have become a major tool in many important results. However, this seminal concept is still not enough to cope with some effective problems: for instance, giving a bound on the maximum number of blowing-ups to be performed on a surface before its multiplicity decreases. This short note shows why such a bounding is not possible, at least with the original definitions.

Mathematics Subject Classification (2000): 14H20, 32S25.
\end{abstract}

\section{Introduction}

In this paper we will deal with embedded algebroid surfaces, that is, schemes given by the spectrum of a ring $R=K[[X, Y, Z]] /(F)$, where $K$ is an algebraically closed field and $F$ is a power series of order $n>0$. Such an $F$ will be called an equation of the surface and $n$ will be called the multiplicity of the surface.

Theorem.- (Levi-Zariski, algebroid version) Let $\mathcal{S}$ be an algebroid embedded surface with normal crossing singularities. Then if

*Supported by Universidad de Sevilla and MCyT BFM2000-1523

${ }^{\dagger}$ Supported by FQM 218 and MTM2004-07203-C02-01. 
we sucessively blow up smooth equimultiple subvarieties of maximal dimension, the multiplicity is dropped in a finite number of steps.

The fact that a surface can be resolved by blowing up "maximal" centers was already investigated by Beppo Levi ([]) and actually proved by Zariski (11]) in characteristic zero as a part of his proof of the resolution for three-dimensional varieties. His results and techniques were paralleled by Abhyankar in positive characteristic ([1]).

It was Hironaka, however, who got a massive breakthrough by considering for the first time the use of combinatorial tools in singularity theory $([5,6]$ ), a technique who has made possible to tackle a number of problems (see for instance 2, 8, 9, 4] or, for a much more complete information, the excellent survey [3]).

As for surfaces is concerned, a combinatorial approach to the LeviZariski theorem was pointed out by Hironaka in the introduction of [5], and partially developed in [6], although the arguments are not very clear in the positive characteristic case. The original purpose of this paper was sharing the combinatorial approach, using a somehow different induction argument to produce an upper bound for the number of blowing-ups that can be performed, following the LeviZariski procedure, before the multiplicity drops. In the case of curves, this was attached quite straightforwardly using the first characteristic exponent (see, for instance, [5] and [10]). However, we have found that already for surfaces this bounding is not possible (at least using Hironaka's characteristic polygon).

The authors are enormously grateful to Prof. J.L. Vicente, from whom they learned the subject and who posed this question to the last author some years ago. This paper is dedicated to him on his 1000000th (binary) birthday .

\section{Some technical set-up}

For the sake of completeness, we recall here well-known technical results that will be of some help in the sequel.

Let $\mathcal{S}$ be an embedded algebroid surface of multiplicity $n, F$ an equation of $\mathcal{S}$. After a change of variables, one can take $F$ to the 
(Weierstrass) form

$$
F(X, Y, Z)=Z^{n}+\sum_{k=0}^{n-1} a_{k} Z^{k}, \text { where } a_{k}(X, Y)=\sum_{i, j} a_{i j k} X^{i} Y^{j}
$$

To this situation a combinatorial object may be attached: note

$$
N_{\{X, Y, Z\}}(F)=\left\{(i, j, k) \in \mathbf{N}^{3} \mid a_{i j k} \neq 0\right\} \cup\{(0,0, n)\},
$$

where we will omit the subscript whenever the variables are clear from the context. The Hironaka (or Newton, or Newton-Hironaka,...) polygon of $F$ is

$$
\Delta_{\{X, Y, Z\}}(F)(\text { or } \Delta(F))=\mathrm{CH}\left(\bigcup_{a_{i j k} \neq 0}\left[\left(\frac{i}{n-k}, \frac{j}{n-k}\right)+\mathbf{N}^{2}\right]\right),
$$

where CH stands for the convex hull. This object was already used in the famous Bowdoin notes lectures by Hironaka ([6]) and it appeared in printed form for the first time in the outstanding paper [5], where it covered the surface case of the much more general notion of characteristic polyhedron of a singularity.

Remark.- If we allow $Z$ to vary, using changes of variable of the type

$$
Z \longmapsto Z+\alpha(X, Y), \text { with } \alpha \in K[[X, Y]] \text { not a unit, }
$$

we obtain a collection of polygons which has a minimal element in the sense of inclusion (this is not obvious at all in positive characteristic). This object was called by Hironaka the characteristic polygon of the pair $(\mathcal{S},\{X, Y\})([\underline{5})$, noted $\Delta(\mathcal{S},\{X, Y\})$.

Remark.- For the case of characteristic zero or, more broadly, the case where $n$ does not divide the characteristic of $K$, it is customary to make the Tchirnhausen transformation,

$$
Z \longmapsto Z-\frac{1}{n} a_{n-1}(X, Y),
$$

which is a change of variables of the type considered by Hironaka. Such an equation will be called a WT equation and it has many interesting properties, which we will show. To begin with, in these equations, a permitted (that is, equimultiple and smooth) curve can be written in the form $\mathfrak{p}=(Z, G(X, Y))$. 
Definition.- A vertex $\left(P_{1}, P_{2}\right)$ of $\Delta(F)$ is called contractible if there exists a change of variables $\varphi$

$$
Z \longmapsto Z+\alpha X^{a} Y^{b}, \text { with } \alpha \in K,
$$

such that

$$
\Delta(\varphi(F)) \subset \Delta(F) \backslash\left\{\left(P_{1}, P_{2}\right)\right\} .
$$

Were this the case, $\varphi$ is called the contraction of the vertex $\left(P_{1}, P_{2}\right)$.

Remark.- If we can apply the Tchirnhausen transformation, the resulting equation has no contractible vertices. In fact, a vertex $(a, b)$ is contractible if and only if it represents all the monomials from $\left(Z+\alpha X^{a} Y^{b}\right)^{n}$ and this cannot happen since $a_{n-1}(X, Y)=0$. As it will become obvious from the equations associated to the different blowing-ups, this situation will remain during the resolution process (at least, until a multiplicity decrease happens). In classical terms, $Z=0$ is a linear hypersurface with permanent maximal contact with the surface $\mathcal{S}$.

Hironaka proved in [5] (for arbitrary characteristic) that all the vertices of $\Delta(F)$ are not contractible if and only if $\Delta(F)=\Delta(\mathcal{S},\{X, Y\})$. From the previous remark this is obvious in the case of WT equations.

\section{An interesting example}

We will try to prove that Hironaka's characteristic polygon does not contain enough information in order to bound the resolution process, even if we are interested only in a first multiplicity decreasing.

To see that, assume that $K$ has characteristic other than 3 and consider the surface $\mathcal{S}$ defined by the equation

$$
F=Z^{3}+X^{m} Z+(X-Y)^{4}, \text { with } m \geq 19 ;
$$

which is, obviously, a WT equation and, hence, the characteristic polygon of $\mathcal{S}$ is given by 


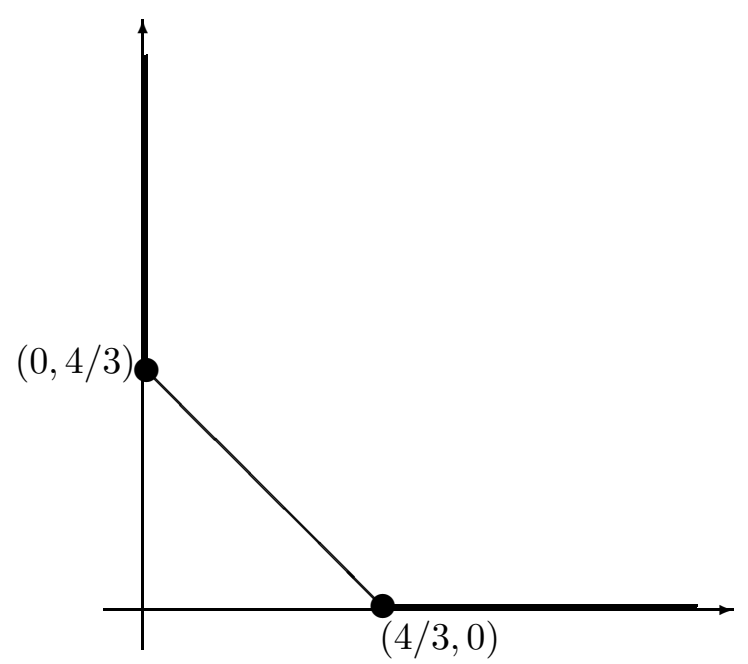

We will first make, in absence of permitted curves, a quadratic transformation (that is, blowing-up the origin) on the direction (1 : $1: 0)$, giving as a result the surface $\mathcal{S}_{1}$ defined by

$$
F_{1}=Z^{3}+X^{m-2} Z+X Y^{4} .
$$

Next we will make two quadratic transformations on the direction $(1: 0: 0)$ (no permitted curves in either surfaces) after which we get $\mathcal{S}_{3}$, defined by

$$
F_{3}=Z^{3}+X^{m-6} Z+X^{3} Y^{4} .
$$

We are hence forced now to perform a monoidal transformation (blowing-up of a curve) centered on $(Z, X)$, as it is now permitted. We get then $\mathcal{S}_{4}$, defined by

$$
F_{4}=Z^{3}+X^{m-8} Z+Y^{4}
$$

Now it is straightforward that, after three quadratic transformations centered on $(1: 0: 0)$ and a monoidal transformation center on $(Z, X)$, we should get a surface $\mathcal{S}_{8}$ defined by

$$
F_{8}=Z^{3}+X^{m-16} Z+Y^{4} \text {. }
$$

Obviously this implies that it is not possible to get a bound for the number of blowing-ups needed for decreasing the multiplicity of 
$\mathcal{S}$ : as we change $m$ we get a family of surfaces with the same Hironaka polygon but needing an arbitrarily large number of blowing-ups to get a multiplicity loss.

Remark.- The key for this counterexample is the first quadratic transformation; in fact it is easy to bound a resolution process using uniquely monoidal transformations or quadratic transformations centered in $(1: 0: 0)$ and/or $(0: 1: 0)$ (this is connected with the Weak Hironaka's Polyhedra Game, solved by Spivakovsky in [8]).

However, as this example makes apparent, if we ever want to bound the resolution process we need to be able to track much more complicated relations between our parameters than the ones considered by Hironaka. We hope this example sheds some light in order to tackle this (for us) quite interesting problem.

\section{References}

[1] S.S. Abhyankar, Resolution of singularities of embedded algebraic surfaces. (Academic Press, New York, 1966).

[2] V. Cossart, 'Desingularization in dimension 2', Resolution of surface singularities (eds.: V. Cossart, J. Giraud, M. Hermann). Lecture Notes in Mathematics 1101 (Springer, Berlin, 1984).

[3] H. HAuser, 'Seventeen obstacles for the resolution of singularities', in Singularities (eds.: V.I. Arnold, G.-M. Greuel, J. Steenbrink). Progress in Mathematics 162 (Birkhäuser, Basel, 1998).

[4] H. Hauser, 'Excellent surfaces and their taut resolution', in Resolution of Singularities (eds.: H. Hauser, J. Lipman, F. Oort, A. Quirós). Progress in Mathematics 181 (Birkhäuser, Basel, 2000).

[5] H. Hironaka, 'Characteristic polyhedra of singularities', J. of Math. Kyoto Univ. 7 (1967) 251-293.

[6] H. Hironaka, 'Desingularization of excellent surfaces', Resolution of surface singularities (eds.: V. Cossart, J. Giraud, M. Hermann). Lecture Notes in Mathematics 1101 (Springer, Berlin, 1984).

[7] B. LEvi, 'Risoluzione delle singolarità puntualli delle superficie algebriche', Atti Acad. Sci. Torino 33 (1897) 66-86.

[8] M. Spivakovsky, 'A solution to Hironaka's polyhedra game', Arithmetic and Geometry II. Progress in Mathematics 36 (Birkhäuser, Boston, 1983). 
[9] M. Spivakovsky, 'A counterexample to Hironaka's hard polyhedra game', Publ. Res. I. Mat. Sci. 18 (1983) 1009-1012.

[10] O. Zariski, Algebraic surfaces (Springer, Berlin, 1935).

[11] O. ZARISKI, 'Reduction of singularities of algebraic three dimensional varieties' Ann. Math. 45 (1944) 472-542.

R. Piedra.

Departamento de Álgebra.

Facultad de Matemáticas.

Universidad de Sevilla.

Apdo. 1160. 41080 Sevilla (Spain).

E-mail: piedra@algebra.us.es

J.M. Tornero.

Departamento de Álgebra.

Facultad de Matemáticas.

Universidad de Sevilla.

Apdo. 1160. 41080 Sevilla (Spain).

E-mail: tornero@algebra.us.es 